\title{
Le mouvement étudiant mexicain de 1968 dans la presse régionale d'Oaxaca
}

El movimiento estudantil mexicano de 1968 en la imprensa de Oaxaca

The 1968 Mexican student movement in the Oaxaca Press

\section{Guillaume Duarte}

\section{OpenEdition}

\section{Journals}

\section{Édition électronique}

URL : https://journals.openedition.org/ideas/2709

DOI : 10.4000/ideas.2709

ISSN : 1950-5701

\section{Éditeur}

Institut des Amériques

\section{Référence électronique}

Guillaume Duarte, «Le mouvement étudiant mexicain de 1968 dans la presse régionale d'Oaxaca », IdeAs [En ligne], 11 | 2018, mis en ligne le 18 juin 2018, consulté le 20 octobre 2022. URL : http:// journals.openedition.org/ideas/2709 ; DOI : https://doi.org/10.4000/ideas.2709

Ce document a été généré automatiquement le 20 octobre 2022

\section{(i) $\odot$

Creative Commons - Attribution - Pas d'Utilisation Commerciale - Pas de Modification 4.0 International - CC BY-NC-ND 4.0

https://creativecommons.org/licenses/by-nc-nd/4.0/ 


\title{
Le mouvement étudiant mexicain de 1968 dans la presse régionale d'Oaxaca
}

\author{
El movimiento estudantil mexicano de 1968 en la imprensa de Oaxaca
}

The 1968 Mexican student movement in the Oaxaca Press

\section{Guillaume Duarte}

1 En octobre 1968, alors que le Mexique s'apprête à célébrer les Jeux Olympiques, un mouvement étudiant s'organise à Mexico et dans l'ensemble du pays pour protester contre les violences policières, puis pour exiger une réforme de la Constitution et du système universitaire. L'incapacité du pouvoir fédéral à dialoguer avec les étudiants entraîne une radicalisation du mouvement et sa répression. Il convient de faire une lecture du mouvement étudiant mexicain entre juillet et octobre 1968 depuis son analyse dans la presse de l'état d'Oaxaca.

2 Carteles del Sur, El Imparcial et Oaxaca Gráfico, trois quotidiens conservateurs d'Oaxaca, couvrent la mobilisation. Si les deux premiers cités sont proches des autorités gouvernementales, Oaxaca Gráfico est plus critique envers la ligne politique du pouvoir fédéral durant le mouvement à Mexico. Les journalistes le comparent à celui qui s'organise, dans une moindre mesure, à Oaxaca. Articles et éditoriaux nous renseignent sur le traitement de l'information pendant le conflit et sur la position de la presse vis-àvis des grévistes ainsi que du gouvernement. Il convient donc de s'interroger sur l'interprétation de la mobilisation étudiante de 1968 dans la presse régionale et sur l'image qu'elle entend véhiculer auprès de l'opinion publique.

\section{Défense de l'autonomie universitaire face aux provocateurs}

3 À Mexico, la protestation débute le 23 juillet à la suite de l'intervention de la police anti-émeutes pour réprimer une rixe opposant des élèves d'écoles préparatoires de 
l'IPN (Institut polytechnique national) et de l'UNAM (Université nationale autonome de Mexico), les étudiants manifestent le 26 juillet et affrontent les forces de l'ordre dans le centre historique de la capitale. L'Armée est déployée le 30 juillet pour déloger les protestataires barricadés dans plusieurs facultés de l'UNAM. Elle force l'entrée du palais San Ildefonso au bazooka et procède à de nombreuses arrestations qui poussent le recteur Javier Barros Sierra à dénoncer la violation de l'autonomie universitaire. Il défile aux côtés des étudiants qui s'organisent en comités de lutte et présentent une série de revendications aux autorités fédérales début août ${ }^{1}$.

4 C'est à partir de ces évènements que les quotidiens d'Oaxaca titrent leurs premières unes sur le mouvement. Si les manifestations et les affrontements y sont mentionnés, ce sont surtout l'intervention du président Gustavo Díaz Ordaz depuis Guadalajara et l'intervention militaire dans les locaux universitaires qui retiennent l'attention des journalistes. La conférence présidentielle est interprétée comme un appel à l'unité et une main tendue aux étudiants. Le débat est plus âpre concernant la responsabilité des étudiants dans la violation de l'autonomie de l'UNAM (Carteles del Sur, 3 août). Pour les éditorialistes, le statut de l'université est menacé par des agitateurs "anarchocommunistes » mexicains et étrangers infiltrés dans les rangs étudiants dans le but de discréditer le pays à l'approche des Jeux Olympiques (El Imparcial, 7 août). Seul Oaxaca Gráfico parle d'exactions commises par les forces de l'ordre contre les étudiants et accuse la presse de se censurer (Oaxaca Gráfico, 2 août).

5 À Oaxaca, les étudiants et le recteur de l'UABJO (Université autonome Benito Juárez), solidaires de ceux de Mexico, manifestent le 6 août. La presse locale souligne que la marche est pacifique et ordonnée autour du recteur Agustín Márquez Uribe et de la FEO (Fédération étudiante d'Oaxaca). Ceux-ci publient des tribunes pour rappeler, outre leur volonté de défendre l'autonomie universitaire, les menaces que font peser les provocateurs « anti-Mexico » sur la patrie, et les responsabilités des étudiants envers le pays (Oaxaca Gráfico, 8 août). Même si un comité de grève se constitue et que l'université est occupée, les journaux saluent la décision de la FEO de ne pas soutenir le $\mathrm{CNH}^{2}$ (Consejo nacional de huelga). Par voie de presse, la fédération étudiante rassure l'opinion publique en affirmant limiter son action au cadre universitaire et rester dans la légalité, à l'inverse des « anarchistes » du conseil national de grève (Carteles del Sur, 18 août).

6 Les déclarations de la FEO correspondent à la ligne politique défendue par les autorités fédérales et universitaires, qui cherchent à contenir le mouvement. Pour cela, l'État envoie des agents arrêter les brigades du $\mathrm{CNH}$, dont l'objectif est de rallier les étudiants de province à leur cause, et les recteurs appellent à reprendre les cours. Cependant, à Mexico, les actions de protestation se multiplient : les établissements sont occupés et d'importantes marches sont organisées vers la principale place de la capitale, le Zócalo. Les correspondants des quotidiens d'Oaxaca à Mexico parlent d'une ambiance « irrespirable » où des « agitateurs » propagent des rumeurs de massacres d'étudiants pour semer le "chaos" (Carteles del Sur, 16 août). Leur traitement des heurts, qui découlent de la manifestation du 27 août, en est l'exemple : alors que la marche est qualifiée d'ordonnée malgré des slogans injurieux contre le président (Carteles del Sur, 28 août), le lendemain, des «agitateurs professionnels " sont accusés d'outrage à la Nation. Ces derniers ont en effet remplacé le drapeau national qui trône au milieu du Zócalo, où a convergé le cortège, par celui de la grève, avant d'être délogés par l'Armée (Carteles del Sur, 29 août). 
7 Bien que le $\mathrm{CNH}$ multiplie les communiqués de presse pour appeler au calme et au dialogue (El Imparcial, 30 aout), les journaux d'Oaxaca sont virulents à l'encontre des étudiants considérés comme des "privilégiés " et désapprouvés par la société. Le discours annuel du président devant le Congrès du Mexique est attendu pour clarifier la position de l'exécutif face à la protestation.

\section{Soutiens au président et à sa politique}

Gustavo Díaz Ordaz présente son quatrième rapport présidentiel le $1^{\mathrm{er}}$ septembre. La presse d'Oaxaca se montre dithyrambique à son encontre, elle salue son " patriotisme " et son «formidable impact sur l'opinion publique » ainsi que sa fermeté à l'encontre des grévistes et son appel au respect de l'ordre juridique (Carteles del Sur, 2 septembre). Les journalistes affirment que si sa main tendue au début du conflit a été perçue comme un aveu de faiblesse, dorénavant le président n'hésitera pas à recourir aux forces armées pour garantir la sécurité du pays. Son intransigeance tend à clarifier une situation que la presse considérait comme pesante du fait du silence institutionnel. La presse d'Oaxaca le présente également comme ouvert au dialogue: il promet une réforme éducative, le respect de l'autonomie universitaire et la révision des pétitions des grévistes (Oaxaca Gráfico, 2 septembre). L'analyse du discours présidentiel par les éditorialistes sert aussi à discréditer le mouvement auprès de l'opinion publique. Ils rappellent que le président n'a reçu aucune pétition du CNH et qu'il est soutenu par les partis politiques, les syndicats, les législateurs et l'Armée (El Imparcial, 3 septembre). Pour la presse régionale, les bases pour la résolution du conflit sont posées.

9 Cependant, à Oaxaca, la ligne politique présidentielle divise les protestataires. Lors d'un meeting du comité de grève, la FEO est critiquée pour ne pas avoir soutenu le $\mathrm{CNH}$ à ses débuts. Puis, après un hommage au drapeau national rendu par des étudiants à l'UABJO, celle-ci est occupée par un groupe armé dissident. Après cet évènement, considéré comme une atteinte à l'autonomie universitaire, les grévistes demandent l'expulsion d'étudiants membres du comité de grève pour avoir «trahi le mouvement» ( $E l$ Imparcial, 9 septembre). Différents groupes d'étudiants de l'UABJO prennent position dans la presse par rapport à la mobilisation étudiante et au gouvernement. Ils rappellent que le comité de grève d'Oaxaca a été formé en soutien à celui de Mexico, qu'il ne lutte pas contre les institutions fédérales mais qu'il a depuis été utilisé par des étudiants «marxistes» pour s'attaquer à la patrie et faire pression sur le gouvernement d'Oaxaca (Carteles del Sur, 13 septembre).

Si la presse et une majorité des étudiants d'Oaxaca soutiennent le président, la violence avec laquelle son gouvernement réprime le mouvement protestataire fait débat.

\section{Répression fédérale d'un mouvement subversif}

11 La presse d'Oaxaca s'inquiète de la tournure que prend le conflit lors de l'occupation de la cité universitaire de l'UNAM par les militaires le 18 septembre. Elle est suivie de celles des campus de l'IPN après plusieurs jours d'affrontements avec les étudiants (Carteles del Sur, 24 septembre).

Le gouvernement est critiqué pour avoir préféré « les baïonnettes au lieu du dialogue » (El Imparcial, 21 septembre). Si les propos du recteur de l'UNAM, qualifiant le 
déploiement des troupes «d'intervention disproportionnée», sont repris, ses détracteurs appellent à sa démission. Des éditorialistes dénoncent l'irresponsabilité des étudiants et d'autres reprochent à la société mexicaine son manque d'autorité morale envers sa jeunesse (Carteles del Sur, 26 septembre). Dans son ensemble, la presse parle de l'urgence d'un retour au calme avant les Jeux Olympiques.

13 Fin septembre, l'IPN puis l'UNAM sont rendus à leurs occupants après que le CNH a annoncé la pacification de ses actions et la réalisation d'un plébiscite pour la reprise des cours. À Oaxaca, les journaux félicitent le conseil universitaire et les étudiants de l'UABJO d'avoir défendu l'institution en étant restés dans la légalité (Carteles del Sur, 29 septembre). Alors que la situation se formalise, les affrontements meurtriers du 2 octobre sur la place des Trois cultures à Tlatelolco sidèrent la presse. Les correspondants qui couvrent un meeting du $\mathrm{CNH}$ relatent qu'un signal lancé depuis des hélicoptères a initié le déploiement des forces armées pour disperser le rassemblement. C'est alors que des francs-tireurs, issus des rangs protestataires et positionnés dans les tours résidentielles autour de la place, ouvrent le feu sur les militaires qui répondent en tirant sur la foule. Qualifiés " d'extrémistes déguisés en étudiants", ils sont pour la presse, qui reprend la version des autorités, les responsables du massacre (Carteles del Sur, 3 octobre). Le discours officiel stipule également que l'Armée est intervenue à la demande de la police métropolitaine et que le bataillon "Olympia " $^{3}$ été envoyé sur la place pour débusquer les tireurs et arrêter les dirigeants du CNH (Oaxaca Gráfico, 3 octobre). Le nombre des victimes oscille entre 6 et 150 morts et entre 100 et 500 blessés, selon les journaux.

Alors que dans la capitale fédérale l'armée quadrille la ville et poursuit l'arrestation des dirigeants du mouvement, à Oaxaca, le comité de grève vote la reprise des activités administratives de l'UABJO et reporte celle des cours à la résolution du conflit ( $E l$ Imparcial, 5 octobre). La presse régionale cesse d'écrire sur le mouvement à Oaxaca, puis sur celui de Mexico, remplacé par l'actualité des Jeux Olympiques inaugurés le 12 octobre. Ces derniers, longtemps défendus, sont désormais critiqués après le massacre des étudiants. Les journalistes reprochent à cet événement sportif d'entraîner la radicalisation de la mobilisation étudiante et le désengagement de l'État fédéral dans le développement de régions telle qu'Oaxaca (Carteles del Sur, 4 octobre). Après avoir voté une trêve durant les Jeux, le $\mathrm{CNH}$ vote la reprise des cours le 4 décembre, avant de se dissoudre et mettre fin au mouvement étudiant de 1968.

La lecture des articles sur le mouvement étudiant de Mexico révèle que la presse d'Oaxaca, malgré la présence de correspondants, reprend les versions officielles du gouvernement pour le décrire. Elle soutient le président dans son approche autoritaire de la protestation et la Nation est présentée comme menacée par des agitateurs politiquement radicalisés. À Oaxaca, le mouvement reste dans la légalité et n'attise guère les critiques des journaux comme s'ils voulaient le contenir et éviter les débordements de Mexico. Les journalistes ne comprennent pas le sens de la protestation estudiantine et seront de nouveau surpris lors des mobilisations des années 1970 où Oaxaca devient un centre des luttes contre le pouvoir dominant. 


\section{BIBLIOGRAPHIE}

PONIATOWSKA, Elena, La noche de Tlatelolco, Mexico, Era, 2015 [1971].

RAMírEz, Ramón, El movimiento estudiantil de México (julio/diciembre de 1968), Mexico, Era, 1998.

02/08/1968 : Oaxaca Gráfico, Oaxaca, « Los estudiantes piden la supresión del cuerpo de granaderos y jefes policiacos », p. 1 et 4 .

03/08/1968 : Carteles del Sur, Oaxaca, « El llamado presidencial », p. 2.

07/08/1968 : El Imparcial, Oaxaca, Juan MARTÍNEZ DE LEÓN, « La autonomía universitaria ».

08/08/1968 : Oaxaca Gráfico, Oaxaca, « Discurso del rector universitario oaxaqueño ».

11/08/1968 : Carteles del Sur, Oaxaca, « Los estudiantes universitarios rechazan toda agitación », p. 3.

12/08/1968 : El Imparcial, Oaxaca, « Rechazo a la agitación ».

14/08/1968 : El Imparcial, Oaxaca, « Ultimátum al politécnico », p. 2.

18/08/1968 : Carteles del Sur, Oaxaca, « Los universitarios oaxaqueños hoy iniciaron su huelga también », p. 1 et 4 .

28/08/1968 : Carteles del Sur, Oaxaca, « Gigantesca manifestación estudiantil ayer en la capital en apoyo de sus peticiones ", p. 1 et 4 .

29/08/1968: Carteles del Sur, Oaxaca, « Acto de desagravio a la bandera nacional en México y control militar de la capital », p. 1 et 4.

30/08/1968 : El Imparcial, Oaxaca, « El estudiantado condena la violencia y pide la solución pacifica del paro », p. 1 et 6 .

01/09/1968 : Carteles del Sur, Oaxaca, Cirilo PANTOJA, « Gran expectación en Oaxaca por el mensaje político del IV informe presidencial hoy », p. 1 et 3.

02/09/1968 : Carteles del Sur, Oaxaca, « Todo tiene su limite dijo el presidente Díaz Ordaz en su enérgico informe de ayer », p. 1 et 4.

02/09/1968 : Oaxaca Gráfico, Oaxaca, « Abre la puerta al dialogo », p. 1 et 4.

03/09/1968 : El Imparcial, Oaxaca, « Apoyo unánime a Díaz Ordaz por el informe que rindió a México ", p. 1 et 6.

09/09/1968 : El Imparcial, Oaxaca, « Armados allanaron por varias horas el edificio central de la máxima casa de estudios », p. 1.

13/09/1968 : Carteles del Sur, Oaxaca, COMITÉ PRO-DERECHOS DEL ESTUDIANTADO OAXAQUEÑO, « Defendamos los derechos básicos del estudiantado oaxaqueño ».

21/09/1968 : El Imparcial, Oaxaca, CANSECO DIAZ Alberto et QUIROGA José Douglas, « Al estudiantado y pueblo en general », p. 2 .

24/09/1968 : Carteles del Sur, Oaxaca, « Feroz batalla entre estudiantes y ganaderos en el POLI anoche", p. 1 .

26/09/1968 : Carteles del Sur, Oaxaca, « La autoridad moral anda mal ». 
29/09/1968 : Carteles del Sur, Oaxaca, « Consejo Nal. de huelga buscara soluciones pacíficamente », p. 1 et 4 .

03/10/1968 : Carteles del Sur, Oaxaca, « Otra vez choques entre estudiantes y el ejército en México: hubo varios muertos », p. 1 .

03/10/1968 : Oaxaca Gráfico, Oaxaca, « Horas trágicas en la metrópoli: batalla en la plaza de las 3 culturas centenares de muertos y heridos », p. 1 .

04/10/1968 : Carteles del Sur, Oaxaca, « ¿Todo por la olimpiada? ».

05/10/1968 : El Imparcial, Oaxaca, « Relativa calma hubo ayer en la capital tras la balacera », p. 1.

\section{NOTES}

1. Pour plus d'informations sur le mouvement étudiant de 1968 au Mexique voir : Poniatowska, Elena, La noche de Tlatelolco, Mexico, Era, 2015 [1971] et Ramírez, Ramón, El movimiento estudiantil de México (julio/diciembre de 1968), Mexico, Era, 1998.

2. Les comités de luttes des institutions en grève (IPN, UNAM, université de Chapingo) se rassemblent autour du $\mathrm{CNH}$ le 8 août. Ils demandent la libération des prisonniers politiques, la dissolution du corps de la police anti-émeute ou encore l'abrogation de l'article $145 \mathrm{du}$ Code pénal qui condamne ceux qui menacent la souveraineté de l'État en propageant des idées politiques étrangères.

3. Unité d'élite formée de militaires et de membres des services secrets chargés de la sécurité des Jeux Olympiques. Présents dans la foule le 2 octobre à Tlatelolco, habillés en civil et vêtus d'un gant blanc, ils ouvrent le feu sur les militaires, blessant le général Hernández Toledo responsable des opérations, pour initier la répression et arrêter les dirigeants étudiants.

\section{AUTEUR}

\section{GUILLAUME DUARTE}

Guillaume Duarte, doctorant en histoire à l'IHEAL (Institut des Hautes Etudes de l'Amérique latine), Université Sorbonne Nouvelle - Paris 3. Membre du CREDA et doctorant associé au CEMCA. Précédemment doctorant contractuel et coordinateur du pôle Mexique de l'IdA (Institut des Amériques). Travaille sur l'histoire sociale des territoires triqui à Oaxaca au $\mathrm{XX}^{\mathrm{e}}$ siècle et les stratégies d'interactions de leurs élites communautaires avec l'Etat mexicain.guillaume.duarte13@gmail.com 